\title{
APPARATUS FOR THE DETERMINATION OF THE MAGNETIC PROPERTIES OF SHORT BARS.
}

By M. F. Fischer.

ABSTRACT.

This paper describes the apparatus and method developed for the magnetic testing of single specimens ro $\mathrm{cm}$ long and $0.6 \mathrm{~cm}$ in diameter. Some types of material, especially those of pure or experimental composition, are available only in small quantities. In the study of correlated properties the work of preparation and heat treatment is minimized if the sample is small.

As the magnetic properties of small samples are difficult to measure directly, a method was adopted which involves the comparison of the test sample with a reference bar whose properties have been previously determined by calibration with several standard bars. The accuracy attainable with this method is entirely satisfactory, the errors in most cases being less than 5 per cent.

\section{CONTENTS.}

I. Introduction. ..................

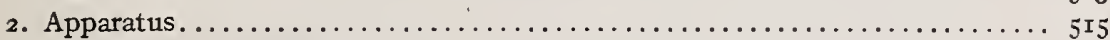

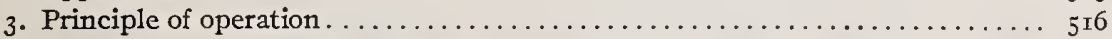

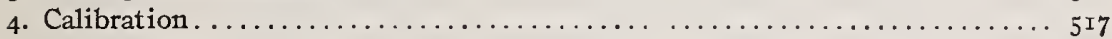

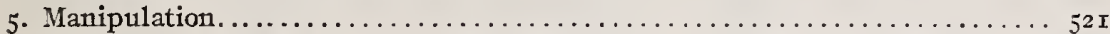

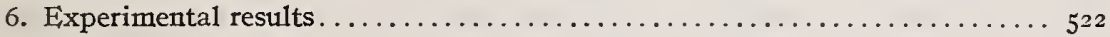

7. Summary............................................ 525

\section{INTRODUCTION.}

In many cases it is desirable to determine the magnetic properties of materials which are available only in small quantities, such as pure alloys, alloys of experimental composition, and the like. For the correlation of the magnetic properties with various factors, such as composition and heat treatment, it would be a distinct advantage if only small samples were required. The ordinary methods of magnetic testing, however, require relatively large samples, so that a method for the testing of small samples with satisfactory accuracy and speed would greatly facilitate such work. In order to meet this need, an apparatus has been developed which is capable of testing small samples with a degree of accuracy sufficient for most purposes. Consideration was first given to several methods already available which were designed to measure the $13061^{\circ}-22$ 
magnetic properites of small samples and also to some others which might be adapted to this purpose.

The "isthmus method" of Ewing and Low ${ }^{1}$ and the modifications by Campbell and Dye ${ }^{2}$ and Cheney ${ }^{3}$ use samples of small size, but are designed, primarily, to measure magnetic properties corresponding to high values of magnetizing forces and are not sufficiently accurate at the lower values. The double length double yoke method of Ewing ${ }^{4}$ can be used for small specimens, but has the disadvantage that it requires not only two similar specimens but two complete sets of observations for a single induction curve. The permeability bridge of Ewing ${ }^{5}$ and its modifications lack sensitivity, and, furthermore, it is practically impossible to obtain hysteresis data with them.

Experiments were made with small models of the Hopkinson ${ }^{\circ}$ single yoke and the Fahy ${ }^{7}$ simplex permeameter, but the results were not sufficiently promising to warrant further development. The errors for some types of material were large, and no satisfactory corrections could be found that were applicable to all classes of material.

The compensated double yoke method of Burrows ${ }^{8}$ is capable of yielding highly accurate results when the length of the sample is not less than $25 \mathrm{~cm}$, but for samples as short as $10 \mathrm{~cm}$ the proximity of the joint compensating coils to the middle of the test bar produces an appreciable increment of magnetizing force which is not measured and which is therefore in most cases a source of error too great to neglect. This method was used in the present work, however, in obtaining data on the $35 \mathrm{~cm}$ samples used as standards and where statements of accuracy are made the values obtained by this method are used as the basis of reference.

To avoid as far as possible any of the disadvantages of the abovementioned permeameters, notably the requirement of two samples and the use of compensation, a type involving the comparison of an unknown sample with one whose properties have been previously determined was developed. Because of the principle involved this apparatus will be referred to in the following pages as a comparator. In the preliminary study a sample $10 \mathrm{~cm}$ long and $6 \mathrm{~mm}$ in diameter was arbitrarily selected as of suitable size,

\footnotetext{
1 Ewing and Low, Proc, Roy. Soc., 1887: Phil Trans. $2889 \mathrm{~A}$.

${ }^{2}$ Campbell and Dye, J. Inst. Elec. Eng., 54, p. 35; I9I5.

${ }^{3}$ Cheney, B. S. Sci. Papers, No. $36 \mathrm{r}$; 1920.

${ }^{4}$ Ewwing, J. A., Magnetic induction in iron and other metals, p 362.

5 Ewing, J. A., Magnetic induction in iron and other metals, p. 366.

'Hopkinson, Phil. Trans. Roy. Soc., 176; r885.

7 Fahy, Met. and Chem. Eng., 19; I918.

${ }^{8}$ Burrows, B. S. Sci. Papers, No. Ir7; 1909.
} 
Scientific Papers of the Bureau of Standards, Vol. 18

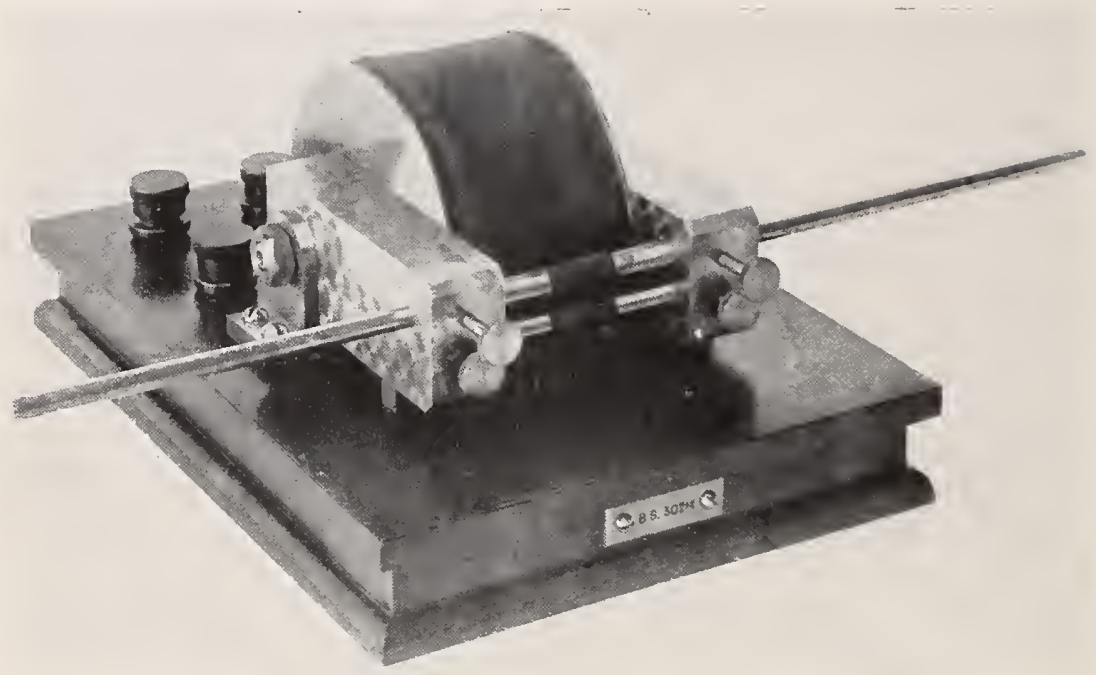

FIG. I - Photograph of magnetic comparator. 
and these dimensions were retained for use in the final form of the apparatus.

\section{APPARATUS.}

Figure I is a photograph of the comparator showing the essential features of its construction. It consists, primarily, of an electromagnet with pole pieces and yoke of Norway iron and a magnetizing solenoid of approximately I,200 turns of No. I 7 copper wire surrounding the yoke. Symmetrically located in the ends of the poles are two $6 \mathrm{~mm}$ holes with centers $\mathrm{I} 2 \mathrm{~mm}$ apart in which the known bar and the bar to be tested are lightly clamped by means of

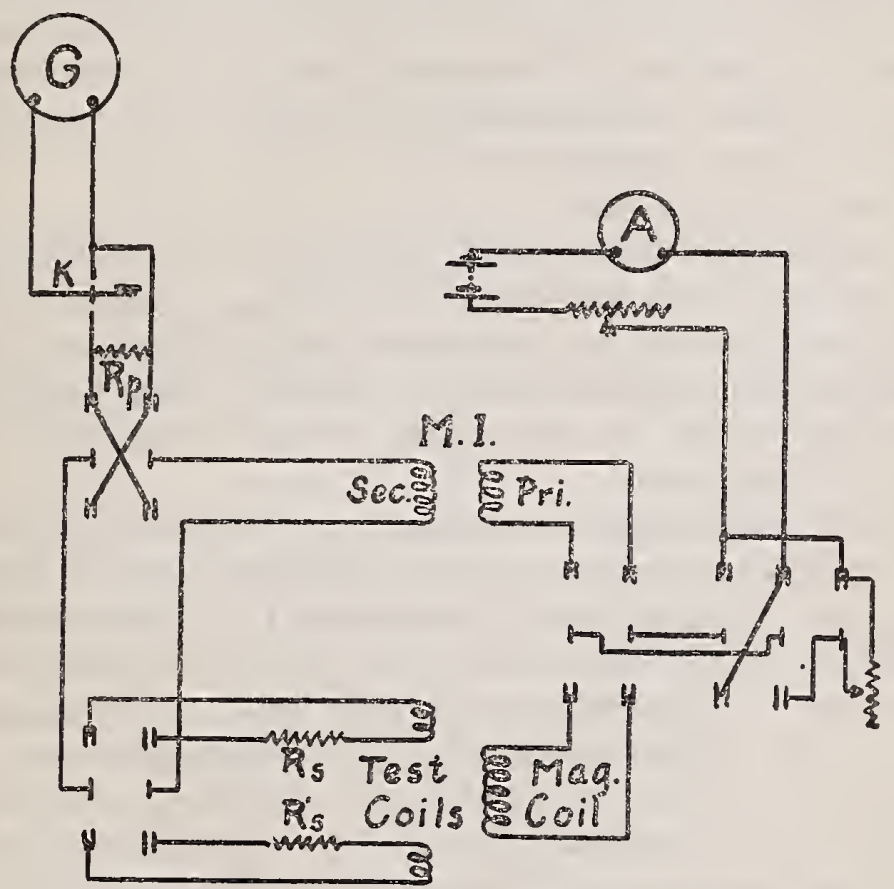

FIG. 2.-Diagram of electrical circuits and connections.

the thumbscrews. Coaxial with these holes and held in position between the poles are two test coils of Ioo turns each, wound on brass forms of a slightly larger internal diameter than the diameter of the bars which they surround. In the photograph is shown a test bar $35 \mathrm{~cm}$ long projecting equal distances beyond the sides of the poles. The other bar is Io cm long, and consequently does not project beyond the sides of the apparatus. The approximate size of the apparatus may be judged from a comparison with the test bar.

The electrical connections are shown in Figure 2. Each of the test coils can be separately connected to the ballistic galvanometer 
through the secondary of the calibrating mutual inductance. The galvanometer is calibrated in the usual way by reversing the proper current through the primary of the mutual inductance and adjusting the sensitivity by means of resistances $R_{s}$ and $R^{\prime}$, so that $\mathrm{I} \mathrm{cm}$ scale deflection corresponds to the reversal of an induction of $\mathrm{I}, 000$ gausses.

\section{PRINCIPLE OF OPERATION.}

In the several permeameters first mentioned the induction is proportional to the quantity of electricity induced in a test coil surrounding the test bar when the magnetizing force is reversed and can be accurately measured by a suitable galvanometer connected to the test coil. . The values of magnetizing force are measured in a manner characteristic of each method and may be considerably in error especially when the values are small or the specimen is as short as $10 \mathrm{~cm}$.

In the present method values of induction are also measured by means of a test coil surrounding the test bar. Values of magnetizing force, however, are obtained by noting the inductions in the second bar, called the reference bar, and from a suitable calibration curve determining the magnetizing forces corresponding to the observed inductions.

If the two specimens were subjected to the same value of magnetizing force for a given value of magnetizing current, the inductions in the two bars would be proportional to their respective permeabilities, and the induction in the reference bar under such circumstances could be used to correctly indicate the magnetizing force. Such a simple procedure is not possible, however, as the reluctance of the joints and leakage from the bars cause discrepancies which are too large to be neglected. This is easy to see when it is remembered that the magnetomotive force drop in the joints is dependent not only on their reluctance, but also on the value of the flux traversing them, which in turn depends upon the permeability of the bar. The situation is further complicated by the fact that with bars of differing permeability the leakage from the two is not the same, and consequently the flux distribution along their length is different. This latter condition causes a different reading according to the length of test coil employed. The best length of test coil to use-approximately $2 \mathrm{~cm}$-was determined by experiment.

While the discrepancies due to the two causes just mentioned are functions of the difference in permeability of the two bars, no 
simple correction factor was found which could be used in computing the true values of magnetizing force. This difficulty has been overcome to a large extent by calibrating the apparatus with standard bars of various properties.

\section{CALIBRATION.}

Since in this method of testing, the inductions in the reference bar are used to indicate values of magnetizing force, it is advisable to select for this purpose a bar whose permeability at the lower values of magnetizing force is relatively high. While this is not absolutely necessary, it is evident that the precision of the results

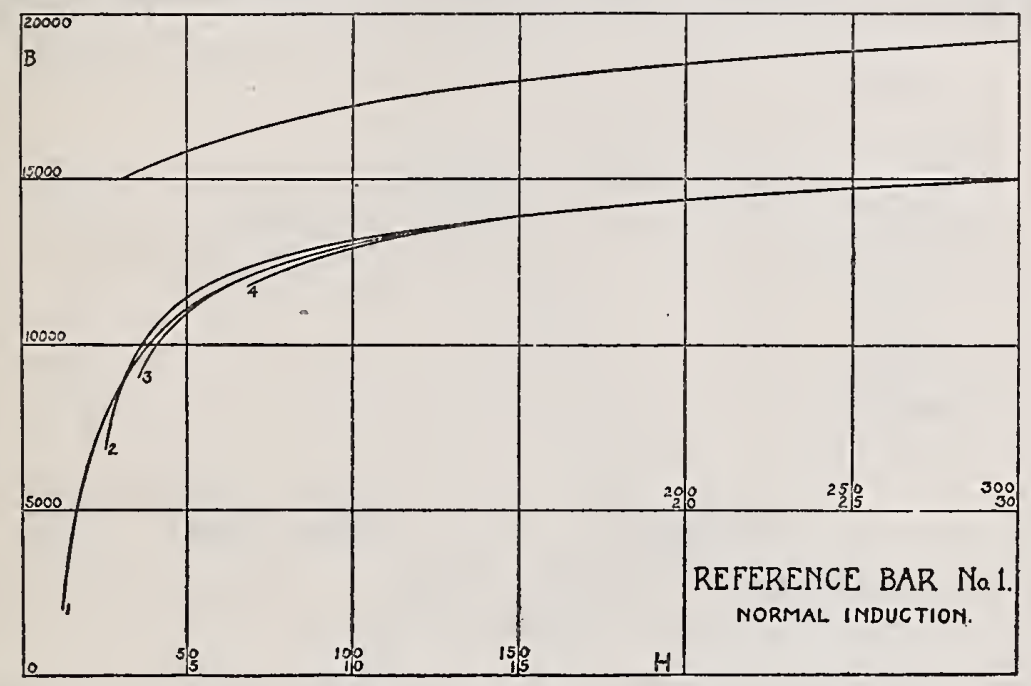

FIG. 3.-Calibration curves for normal induction.

is increased if the inductions in the reference bar for low values of magnetizing force are as high as possible.

Several standard bars $6 \mathrm{~mm}$ in diameter and of a minimum length of $25 \mathrm{~cm}$, each of different magnetic properties, are then tested by the Burrows compensated double-yoke method. The magnetic properties of these bars should range from those corresponding to hardened permanent magnet steel to those representative of such material as annealed pure iron or silicon steel. Each of these bars is then put in the comparator with the reference bar and corresponding values of induction determined for both bars over the range of magnetizing forces usually employed in testing, namely, from the magnetizing force required to produce an induction of 2,000 gausses in the standard bar up to a magnetizing force of 300 gilberts per centimeter. 
The values of magnetizing force in each case are those corresponding to the inductions in the standard bars and are obtained from the previously determined induction curves of the standard bars. With these values of magnetizing force and the observed inductions in the reference bar the apparent induction curve of the reference bar can be drawn.

If it were not for the effect of leakage and the reluctance of the joints, as explained above, the same normal induction curve should be obtained for the reference bar regardless of the properties of the standard used. As this condition is not realized, the curve obtained for values of induction below about I 4,000 gausses depends upon the properties of the bar which is used as a standard. This is illus-

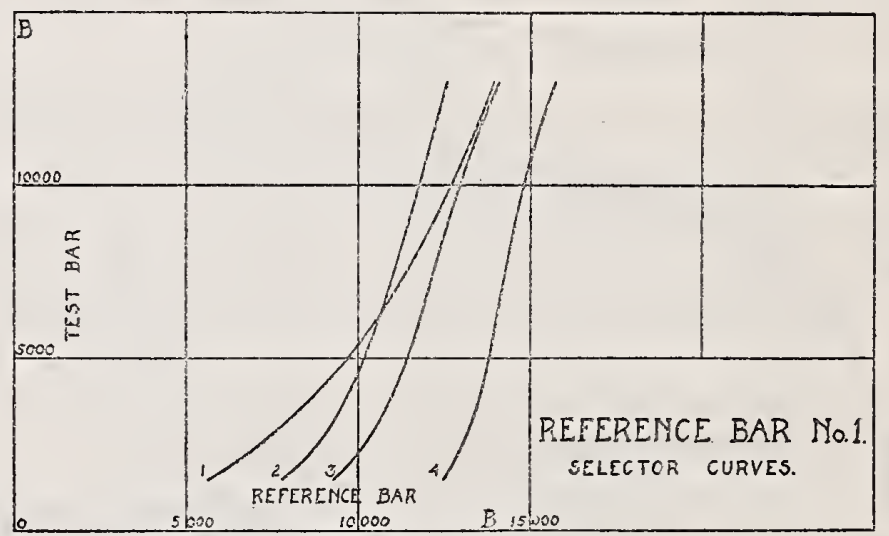

FIG. 4.-Selecior curves for normal induction.

trated in the curves of Figure 3, which were obtained by using standard bars whose properties range from those of magnetically soft material to those of hardened magnet steel. Even with this wide range, however, the lack of agreement between the curves is not very great.

By the use of curves obtained in this manner the errors of the final results are materially reduced. If an unknown bar is substituted for the standard and the corresponding values of induction for the unknown and the reference bar are determined, the curves previously obtained by means of the known standard bars can be used as calibration curves. The seiection of the calibration curve which will give the most accurate results is made by means of curves in which the induction of the standard is plotted against the induction of the reference bar. These curves have been called "selector curves." 
When two or three values of induction of the bar under test are plotted against the corresponding values of induction in the reference bar, the selector curve to which these points come nearest indicates the calibration curve to use. The number of calibration curves necessary depends upon the range of materials to be tested. The curves given in Figure 4 cover a considerable range and are adequate for most purposes. This is demonstrated by the fact that in some cases a single calibration curve is representative of several standard bars whose properties are quite different. For instance curve 4 in Figure 3 is the calibration curve obtained with three standard bars whose properties, as determined by the Burrows method, are indicated by the curves of Figure 5. The cali-

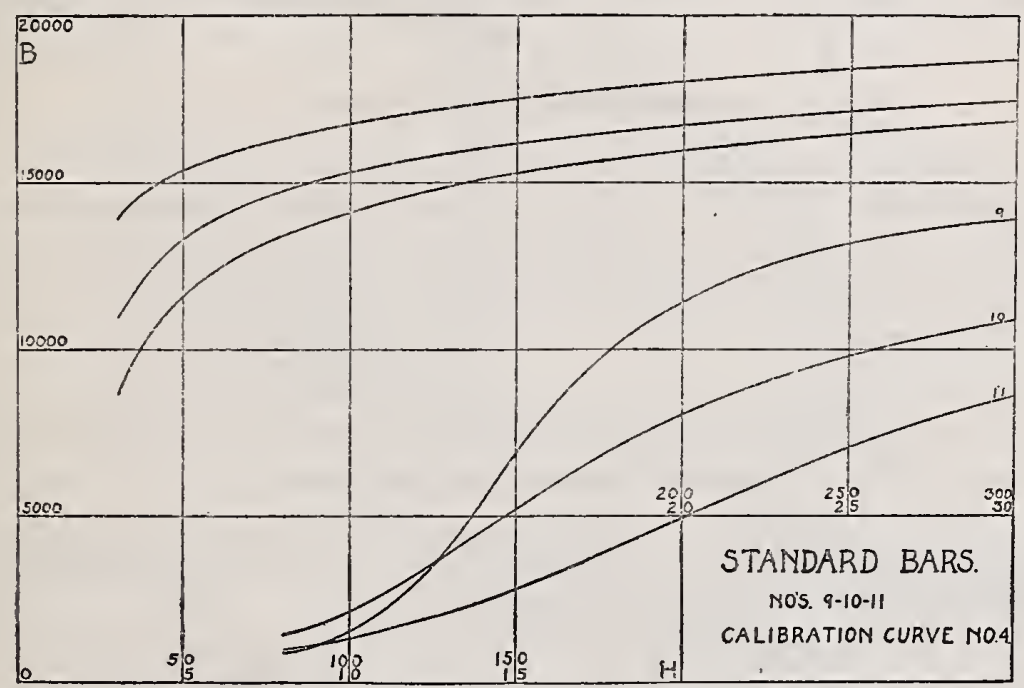

Fig. 5.-Normal induction curves for standard bars 9, IO, and II.

bration curves $\mathrm{I}, 2$, and 3 were obtained by the use of standard bars of magnetically softer material than those used in getting curve 4, but each represents, however, the calibration curve corresponding to two bars of different properties.

It is possible to use this apparatus for the determination of hysteresis values as well as for normal induction. It has been found by experiment that the residual induction can be determined with sufficient accuracy, in general, by merely noting the change in induction when the magnetizing current is reduced to zero. For determining other points on the hysteresis loop, however, a calibration is necessary. It is desirable in this case to use a reference bar having a large coercive force, as it is difficult to obtain readings with sufficient precision with the soft bar used for normal induction. 
The calibration of this bar is also made by comparison with several standard bars whose properties are known from a previous measurement in the Burrows permeameter. The difficulty of preparing a set of standard bars having a wide enough range of coercive forces was overcome by using for most of the points a single bar of magnet steel and by proper heat treatment changing its coercive force to any desired value. Thus, the highest coercive force was observed when this bar was in the quenched condition, and lower values were obtained by successive drawings at increasing temperatures. For coercive forces lower than that of this bar after

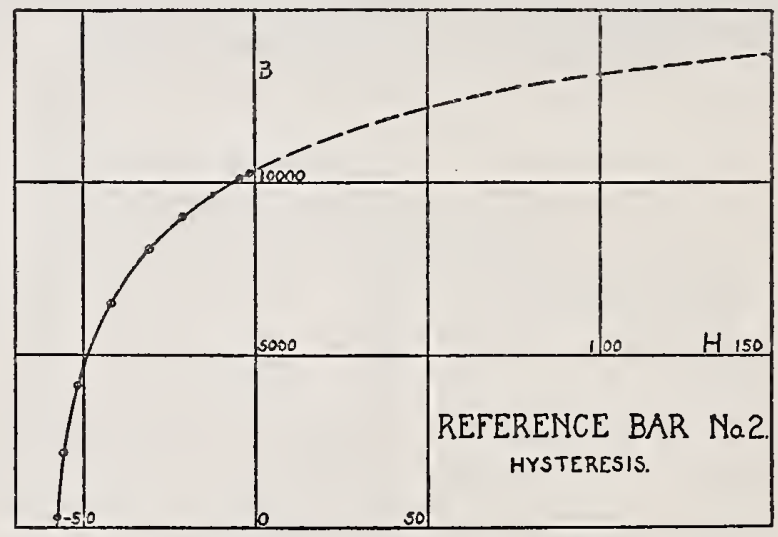

FIG. 6.-Calibration curve for hysteresis.

annealing, several of the standard bars used in the calibration of the first reference bar were employed.

To calibrate this second reference bar, then, it is placed in the comparator with one of the standards. Sufficient magnetizing current is applied to raise the induction in the standard bar to the value corresponding to the magnetizing force from which it is desired to obtain values of residual induction and coercive force. In the present work a maximum magnetizing force of I 50 gilberts per centimeter was used, this being the value most frequently employed in practice. The corresponding induction in the reference bar is used in subsequent testing to indicate a magnetizing force of $H=I_{5} \mathrm{O}$. From this tip a reversed magnetizing force is applied of sufficient magnitude to reduce the induction in the standard bar to zero. This force is the coercive force of the standard bar, and its value is consequently that obtained for the bar by the Burrows method. The application of this reversed magnetizing force does not necessarily reduce the induction in 
the reference bat to zero but to some value which corresponds to the demagnetizing force applied. This induction and the force applied give a point on the calibration curve of the reference bar. Other points are similarly obtained by using standard bars having different coercive forces. The complete calibration curve of this reference bar is that portion of a hysteresis loop beginning at a magnetizing force of zero and extending to a negative magnetizing force whose magnitude is as great as the highest value of coercive force likely to be measured. Figure 6 shows such a calibration curve with points coresponding to each standard used.

In the event that coercive forces corresponding to different values of maximum magnetizing forces are to be obtained, it is necessary that calibrations of the reference bar be made from these same tips.

\section{MANIPULATION.}

The procedure in determining the magnetic properties of unknown material with this apparatus is similar in most respects to that of calibrating the reference bars. The bar to be tested is placed in the comparator through the upper test coil and the reference bar through the lower test coil. After demagnetizing the two bars, the magnetizing current is set to produce in the test bar approximately the minimum induction desired. This induction and the corresponding induction in the reference bar are observed. Similar readings are made for each of successively increasing values of magnetizing force up to the maximum desired. With the present apparatus values of magnetizing force as high as I,O0o gilberts per centimeter can be attained without difficulty.

By referring the first few values of induction to the selector curves some curve will correspond most nearly to that whose coordinates are the values of induction just obtained. The index number of this curve indicates the calibration curve to be used in getting values of magnetizing force. The magnetizing forces are those corresponding to the inductions observed in the reference bar and are consequently the magnetizing forces acting on the test bar. The normal induction curve of the test bar can be drawn from these data.

When values of residual induction and coercive force are to be obtained, the second reference bar is substituted for the first one used. The magnetizing force is adjusted so that its value, as indicated by the induction in the reference bar, is that from which hysteresis values are to be obtained, usually 150 giiberts per 
centimeter. The residual induction is calculated from the observed change in induction in the test bar when the magnetizing current is reduced to zero. In cases where residual induction alone is desired it is not necessary to change reference bars, the value obtained with either bar being sufficiently accurate.

The second reference bar, calibrated for the purpose, must, however, be used in getting coercive force. This is obtained by applying the reversed magnetizing force which will reduce the induction in the test bar to zero. This same force will reduce the induction in the reference bar to some value not necessarily zero. This value of induction will be a point on the hysteresis loop of the reference bar obtained by its calibration, and the corresponding demagnetizing force is the coercive force of the test bar.

\section{EXPERIMENTAL RESULTS.}

To determine with what accuracy measurements could be made, several bars of different magnetic properties were selected and tested both in the Burrows permeameter and in the apparatus herein described. Figure 7 shows curves of the several bars obtained by the Burrows method. The plotted points represent data obtained by means of the comparator. It is to be noted that the plotted points are in good agreement with the curves. The largest errors are found in the lower part of the curves, where the precision of reading is not so good and where the absolute values are generally of less importance. The errors are in general less than 5 per cent of the magnetizing force corresponding to a given induction. Determinations of the properties of the same bars were made by two other methods, designated as I and 2, and are given in Table $\mathbf{I}$. While these other methods are capable of greater accuracy when the material is of larger size, the errors are considerable when the specimens are as small as those used here.

In order to test these bars in the Burrows permeameter, they are necessarily longer than required for the comparator, and consequently project beyond the sides. To determine what effect this projection might have on the accuracy of the results obtained by the comparator, a bar Ioo $\mathrm{cm}$ long was tested and retested after successively decreasing its length. The original bar was placed in the apparatus with its middle portion between the pole pieces, giving a projection on each side of about $45 \mathrm{~cm}$. Each projecting end was in turn cut off, leaving finally a test bar of the 


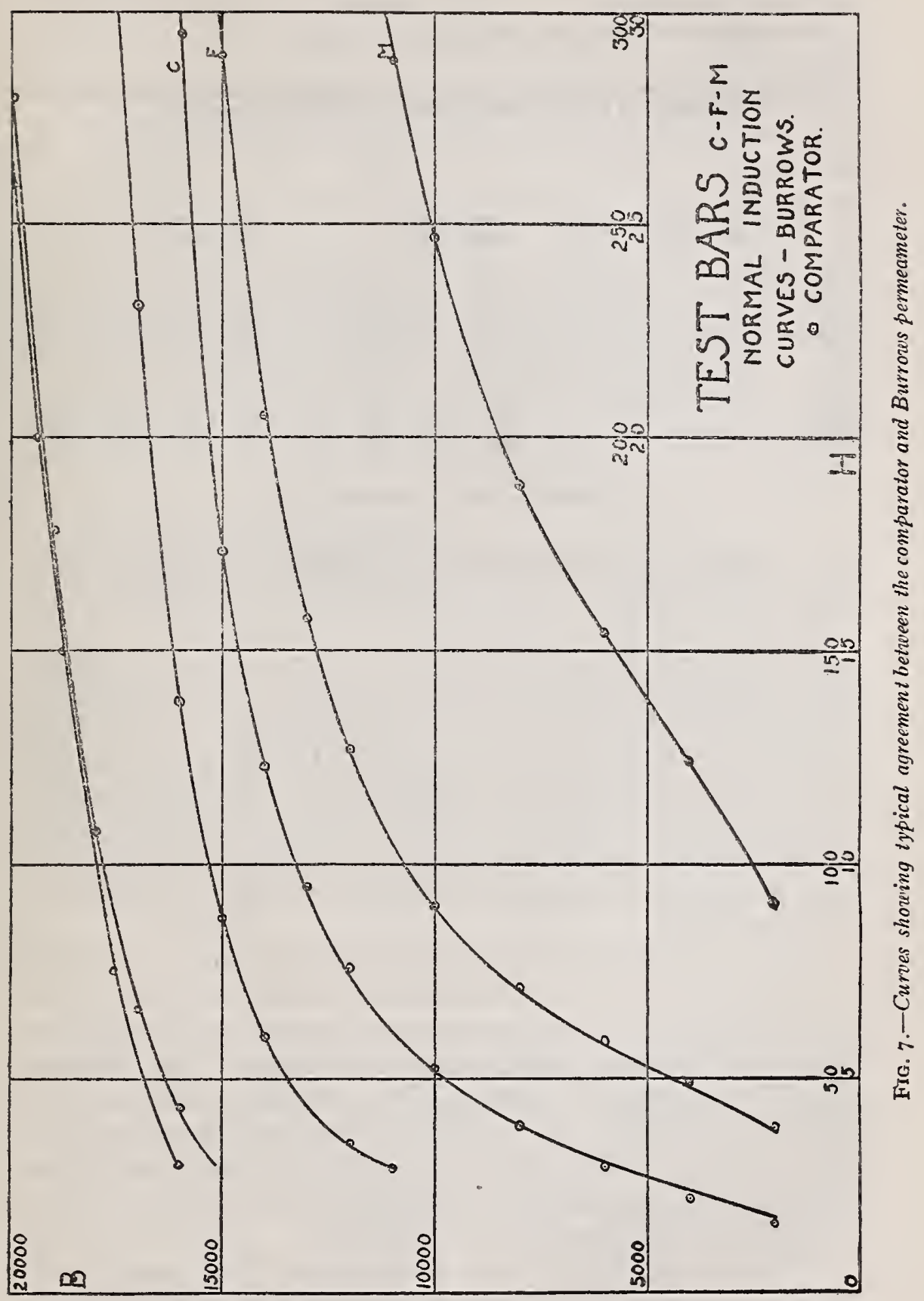


normal length of ro $\mathrm{cm}$, this length being the same portion of the bar tested in each case. The data obtained for the four conditions agree within the limits of observational error.

TABLE 1.-Values of Magnetizing Force Corresponding to Certain Inductions as Obtained by Four Different Methods for Each of Three Bars.

\begin{tabular}{|c|c|c|c|c|c|c|c|c|c|c|c|c|}
\hline \multirow{2}{*}{ Induction. } & \multicolumn{4}{|c|}{ Bar A. } & \multicolumn{4}{|c|}{ Bar B. } & \multicolumn{4}{|c|}{ Bar C. } \\
\hline & $\begin{array}{l}\text { Bur- } \\
\text { rows. }\end{array}$ & $\begin{array}{c}\text { Compa- } \\
\text { rator. }\end{array}$ & 1 & 2 & $\begin{array}{l}\text { Bur- } \\
\text { rows. }\end{array}$ & $\begin{array}{c}\text { Compa- } \\
\text { rator. }\end{array}$ & 1 & 2 & $\begin{array}{l}\text { Bur- } \\
\text { rows. }\end{array}$ & $\begin{array}{c}\text { Compa- } \\
\text { rator. }\end{array}$ & 1 & 2 \\
\hline $\begin{array}{r}2,000 \ldots \ldots . \\
4,000 \ldots \ldots . \\
6,000 \ldots \ldots \\
8,000 \ldots . \\
10,000 \ldots \ldots\end{array}$ & $\begin{array}{l}9.10 \\
12.4 \\
15.4 \\
18.9 \\
24.7\end{array}$ & \begin{tabular}{r|}
9.10 \\
12.4 \\
15.4 \\
18.9 \\
24.5
\end{tabular} & $\begin{array}{l}8.33 \\
11.7 \\
14.7 \\
18.4 \\
23.4\end{array}$ & $\begin{array}{l}8.50 \\
11.5 \\
14.3 \\
17.9 \\
23.3\end{array}$ & $\begin{array}{l}3.74 \\
4.78 \\
5.80 \\
7.03 \\
9.00\end{array}$ & $\begin{array}{l}3.83 \\
4.93 \\
5.85 \\
7.08 \\
9.05\end{array}$ & $\begin{array}{r}3.94 \\
5.35 \\
6.55 \\
8.01 \\
10.3\end{array}$ & $\begin{array}{l}3.69 \\
4.74 \\
5.74 \\
6.98 \\
9.05\end{array}$ & $\begin{array}{l}1.76 \\
2.39 \\
3.00 \\
3.85 \\
5.17\end{array}$ & $\begin{array}{l}1.60 \\
2.15 \\
2.90 \\
3.85 \\
5.26\end{array}$ & $\begin{array}{l}1.82 \\
2.63 \\
3.46 \\
4.50 \\
5.95\end{array}$ & $\begin{array}{l}1.52 \\
2.06 \\
2.63 \\
3.44 \\
4.68\end{array}$ \\
\hline $\begin{array}{l}12,000 \ldots \ldots \\
14,000 \ldots \ldots \\
16,000 \ldots \ldots \\
18,000 \ldots . \\
20,000 \ldots\end{array}$ & $\begin{array}{r}34.6 \\
59.7 \\
137\end{array}$ & $\begin{array}{c}35.2 \\
60.2 \\
138\end{array}$ & $\begin{array}{c}32.7 \\
52.8 \\
102\end{array}$ & $\begin{array}{c}32.8 \\
58.0 \\
136\end{array}$ & $\begin{array}{l}12.6 \\
20.7 \\
42.3 \\
108 \\
280\end{array}$ & $\begin{array}{c}12.7 \\
20.4 \\
43.2 \\
106 \\
285\end{array}$ & $\begin{array}{c}14.0 \\
21.2 \\
39.0 \\
85.0 \\
176\end{array}$ & $\begin{array}{l}12.7 \\
20.5 \\
43.0 \\
113 \\
289\end{array}$ & $\begin{array}{c}7.42 \\
12.3 \\
29.0 \\
103 \\
268\end{array}$ & $\begin{array}{c}7.65 \\
12.3 \\
29.6 \\
94.0 \\
265\end{array}$ & $\begin{array}{c}8.33 \\
12.5 \\
25.6 \\
73.0 \\
162\end{array}$ & $\begin{array}{c}6.81 \\
11.3 \\
27.4 \\
96.0 \\
253\end{array}$ \\
\hline
\end{tabular}

HYSTERESIS VALUES FROM $\mathrm{H}_{\max }=150$.

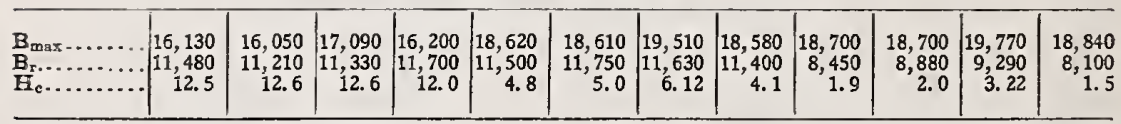

To determine what effect iron in the vicinity of the apparatus might have on its accuracy, a bar was tested under normal conditions and retested with a bar of soft iron $\mathrm{I}$ by $3 \mathrm{~cm}$ in cross section placed directly across the poles of the apparatus, forming a shunt path to the test and reference bars. While this is an extreme condition and unlikely to occur in actual practice, the results obtained in the two cases are identical. The only apparent effect is to increase the magnetizing current necessary to produce a certain induction.

While slight variations from the specified diameter of $6 \mathrm{~mm}$ can be accounted for in the calculations, it is advisable to keep variations within I or 2 per cent, as variations greater than this increase the contact reluctance, and thus increase the errors. No difficulty should be experienced, however, in machining a test bar to a diameter of $6 \mathrm{~mm}$. The consistency with which results on the same bar can be repeated is exceptionally good. Differences obtained on several readings, between each of which the test bar was removed and reinserted, are negligible.

It has been found that bars of commercial steel usually submitted for magnetic tests have such variations in magnetic properties along their length that it is impossible to state how ac- 
curately the observed properties of the test bar are representative of the material of which it is a sample. Where the bar is of sufficient length to permit the separate testing of adjacent sections, the presence of inhomogeneities in any one section is sometimes sufficient to produce a difference of 20 per cent or more between the value of magnetizing force observed for a definite induction in one section of a bar and that observed for the same induction in an adjoining section. Differences in the results obtained by several methods, therefore, can not always be attributed entirely to inaccuracies inherent in any one method. The present method is capable of making measurements which show errors averaging less than 5 per cent of the magnetizing force required for a certain induction. For certain types of material errors greater than this may occur for low values of induction, but for ordinary materials the present accuracy is satisfactory. In any case the accuracy is determined by the degree to which the properties of the standard bar used in the determination of the calibration curve approximate those of the bar under test.

Calibration to cover the whole range of magnetic possibilities to a high degree of accuracy would entail a considerable amount of work. In general, this is not necessary. The set of curves illustrated in Figure 3 are sufficient for most purposes. The calibration need be checked only at infrequent intervals, and then only at a sufficient number of points to be sure that the properties of the reference bars have not changed.

Experiments were made to discover the effect on the accuracy of increasing the distance between pole pieces, thus requiring a longer sample. This procedure brought the calibration curves nearer together, and for a length of sample as great as $25 \mathrm{~cm} \mathrm{a}$ single curve could be used with satisfactory results.

\section{SUMMARY.}

In order to meet the need for a method and apparatus for determining the magnetic properties of small samples of material at moderate values of magnetizing force, apparatus has been designed and constructed which gives satisfactory results. The direct determination of the magnetic properties of small samples is very difficult. For this reason the method adopted is the comparison of the sample with another whose properties can be measured by the ordinary methods.

After proper calibration this method is capable of yielding satisfactory results at good speed with cylindrical samples $10 \mathrm{~cm}$ 
long and $6 \mathrm{~mm}$ in diameter. Errors need not exceed 5 per cent of the magnetizing force for a given induction. It is believed that this method will prove useful in many investigations where limited quantities of material are available or where for any reason small samples are desirable.

R. L. Sanford, of the magnetic laboratory, gave helpful suggestions during the development of this apparatus, and J.M. Barry made many of the observations.

Washington, August i8, 1922. 